TOBIAs CONRADI, HEIKE DERWANZ, FLORIAN MUHLE

\title{
STRUKTURENTSTEHUNG DURCH VERFLECHTUNG - ZUR EINLEITUNG
}

Die Akteur-Netzwerk-Theorie (ANT) fordert, die Verflechtungen zwischen Natur, Kultur, Gesellschaft und Technik neu zu erforschen. Sie bietet einen Blick auf Formen der Vernetzung, die in ungeplante Strukturen münden. Dieser stand im Zentrum des Workshops „Strukturentstehung durch Verflechtung", der im Mai 2010 am Graduiertenkolleg Automatismen an der Universität Paderborn stattfand. Auf diesen Workshop geht der vorliegende Sammelband zurück, in dem nach Verbindungen zwischen Akteur-Netzwerk-Theorie(n) und Automatismenforschung gesucht wird und der an aktuelle Diskussionen über die ANT in den Sozial- und Kulturwissenschaften anschließt. Forschende der unterschiedlichsten Disziplinen nehmen seit einigen Jahren die aus den Science and Technology Studies (STS) kommende ANT zum Anlass, das eigene Fach und die etablierten Forschungsansätze neu zu befragen. Auffällig an der Konjunktur der ANT in der deutschsprachigen Kultur- und Sozialwissenschaft scheint dabei zunächst die Ungleichzeitigkeit: In der englischsprachigen Debatte wurde bereits in den 1980er Jahren kontrovers über die ANT diskutiert. In der Zwischenzeit hat sich das Feld ausdifferenziert und seit mittlerweile mehr als zehn Jahren wird über Projekte nach der ANT nachgedacht. ${ }^{1}$ Die bisherigen deutschsprachigen Publikationen zum Thema enthalten vor allem konzeptionelle Überlegungen und grundlegende Kritiken an der ,klassischen' ANT. ${ }^{2}$

Der Schwerpunkt dieses Bandes ist hingegen anders gelagert. Die BeiträgerInnen $^{3}$ gehen der Frage nach, welchen ,Mehrwert' die ANT speziell für sozial- und kulturwissenschaftliche Fragestellungen bietet. An die Stelle von strikten Abgrenzungen, an denen die eigene Forschungspraxis profiliert wird, tritt so die Suche nach möglichen Anschlüssen und Kompatibilitäten. Der Blick wird hierzu in zwei Richtungen gewendet: Auf der einen Seite steht die Frage, ob und inwieweit die ANT mit etablierten Theorieangeboten kompatibel ist und auf der anderen Seite, was die ANT anderen Theorieangeboten der

Vgl. John Law/John Hassard (Hg.), Actor Network Theory and After, Oxford (u. a.), 1999.

2 Vgl. Georg Kneer/Markus Schroer/Erhard Schüttpelz (Hg.), Bruno Latours Kollektive. Kontroversen zur Entgrenzung des Sozialen, Frankfurt/M., 2008 sowie die Debatte in Sebastian Gießmann/Ulrike Brunotte/Franz Mauelshagen/Hartmut Böhme/Christoph Wulf (Hg.), Zeitschrift für Kulturwissenschaften. Politische Ökologie, Bielefeld, 2 (2009).

3 Wir haben uns innerhalb des gesamten Bandes für den Gebrauch des Binnen-I entschieden. Der Begriff des ,Akteurs` wird als geschlechtsneutrale Kategorie behandelt. 
Kultur- und Sozialwissenschaften verdankt - auch wenn sie dies nicht immer explizit macht.

Vor diesem Hintergrund ist ein Ziel des Bandes, an die Diskussionen über die ANT anzuknüpfen und sie fortzusetzen. Ganz im Sinne der ANT geht es darum, Verbindungen nachzuzeichnen und Kontroversen auszutragen. Damit ist die Hoffnung verbunden, wenn auch nicht zu einer vollkommen neuartigen Forschungspraxis, so doch zu einem verbesserten Verständnis der vielfältigen Verflechtungen zwischen Menschen und Nicht-Menschen zu gelangen. Vielleicht eröffnet sich auf diese Weise auch die Möglichkeit, den Automatismen näher auf die Spur zu kommen, die im Verständnis des Paderborner Graduiertenkollegs als „Bestandteil eines wirkmächtigen Arrangements von Dingen, Zeichen und Subjekten ${ }^{\text {"4 }}$ begriffen werden.

Die Beiträge des Bandes entstammen den verschiedensten Gebieten: von der Soziologie, Geschichtswissenschaft, den Medien- und Kunstwissenschaften bis hin zur Kulturanthropologie und Pflegewissenschaft. Damit spiegelt der Band das breite Interesse wider, das die ANT über disziplinäre Grenzen hinaus auf sich gezogen hat. Gleichzeitig ist an der resultierenden Multidisziplinarität deutlich erkennbar, dass die ANT ihren Nutzen gerade dann entfaltet, wenn ihre theoretischen Prämissen nicht einfach nur ,angewendet' werden, sondern Überlegungen aus der ANT in die Einzeldisziplinen übersetzt und dort mit anderen Angeboten verflochten werden.

Aus dieser Perspektive erscheint die ANT als ein Werkzeugkoffer, der sowohl grundsätzliche kultur- und sozialtheoretische wie auch methodologische und methodische Instrumente enthält, die - im Laufe der vergangenen 30 Jahre - in einer Vielzahl empirischer Fallstudien entwickelt und stetig ausgeweitet wurden. ${ }^{5}$ Hierbei rückt auch in den Blick, dass die Rede von der einen Akteur-Netzwerk-Theorie eine unzulässige Verkürzung darstellt. Die deutschsprachige Debatte scheint in diesem Punkt allzu stark an Bruno Latour orientiert. ${ }^{6}$ Vor diesem Hintergrund haben wir bereits im Titel des Bandes auf die Heterogenität und Fragmentierung unterschiedlicher Ausprägungen der Akteur-Netzwerk-Theorie(n) aufmerksam machen wollen. Damit ist ein weiteres Anliegen dieses Bandes verbunden: die Vielfältigkeit der unter der Bezeichnung (Post-)ANT versammelten Ansätze zu betonen und die Fokussierung auf Latour zu überwinden.

4 Hannelore Bublitz/Roman Marek/Christina L. Steinmann/Hartmut Winkler, „Einleitung“, in: dies. (Hg.), Automatismen, München, 2010, S. 9-16: 10.

Vgl. den Beitrag von John Law in diesem Band.

6 Eine Ausnahme bildet der von Andréa Belliger und David J. Krieger herausgegebene Sammelband ANThology, der Übersetzungen ,klassischer' Texte von Bruno Latour, John Law, Michel Callon und Madeleine Akrich enthält. Andréa Belliger/David J. Krieger (Hg.), ANThology. Ein einführendes Handbuch zur Akteur-Netzwerk-Theorie, Frankfurt/M., 2006. 


\section{Strukturentstehung, ANT und Automatismen}

Während der Terminus des ,Akteur-Netzwerks` zunächst noch Konnotationen einer gewissen Festigkeit oder Stabilität transportiert, deutet eine andere Bezeichnung der ANT - „Soziologie der Assoziationen“7 - darauf hin, dass Strukturen in der Konzeption der ANT niemals etwas abschließend Erreichtes darstellen. ${ }^{8}$ Es geht der ANT in erster Linie immer um die Beobachtung und Beschreibung von Strukturen in ihrem Vollzug. ${ }^{9}$ Hierin sehen wir einen Anknüpfungspunkt, um die Diskussion der ANT mit Fragen nach Automatismen in Beziehung zu setzen. Dieses Anliegen kommt im Titel des Bandes „Strukturentstehung durch Verflechtung“ zum Ausdruck.

Das Konzept der Automatismen, im Sinne des Graduiertenkollegs, geht über seine alltagssprachliche Bedeutung hinaus. Deutlich wird dies insbesondere im Untertitel des Paderborner Kollegs - „Strukturentstehung außerhalb geplanter Prozesse": Automatismen bezeichnen hier nicht eine schlichte Identität der Wiederholung oder das Mechanische des Automaten. ${ }^{10}$ Das Konzept zielt vielmehr auf eine Modellierung von Situationen, in denen die Herausbildung neuartiger, überraschender Strukturen beobachtet werden kann, ohne dass diese auf bewusst und intentional handelnde Instanzen zurückgeführt werden könnten. Die Frage nach den Automatismen wird somit zu einem „Entwicklungsmodell“"11, in dessen Zentrum die Emergenz von Strukturen steht, die durch eine Vielzahl von Akteuren hervorgebracht werden. Die ANT kann hier mit ihrer Fokussierung auf die Rolle nicht-menschlicher Entitäten den Blick auf die Heterogenität der beteiligten Akteure schärfen.

Daneben lassen sich weitere strukturelle Ähnlichkeiten herausstellen. Wenn Automatismen als „Handlungen, die auf spezifische Weise undurchsichtig sind $^{\text {“12 }}$, verstanden werden, dann scheint mindestens die ,Fragerichtung' der ANT der nach Automatismen zu ähneln, wie sich beispielhaft an Latours ganz ähnlich klingender Definition der Handlung deutlich machen lässt:

Handeln ist nicht transparent, es steht nicht unter der vollen Kontrolle des Bewußtseins. Diese altehrwürdige Quelle der Unbestimmtheit ist es, die wir mit dem seltsamen Ausdruck Akteur-Netzwerk wieder lebendig machen wollten: Handeln ist ein Knoten, eine Schlinge, ein Konglomerat aus vielen überraschen-

7 Bruno Latour, Eine neue Soziologie für eine neue Gesellschaft. Einführung in die AkteurNetzwerk-Theorie, Frankfurt/M., 2007 [engl. OA 2005], S. 23.

8 Für alternative Bezeichnungen der ANT vgl. ebd., S. 24.

9 Vgl. zum Prinzip der ,freien Assoziation“ Michel Callon, „Einige Elemente einer Soziologie der Übersetzung: Die Domestikation der Kammmuscheln und der Fischer der St. BrieucBucht" [engl. OA 1986], in: Andréa Belliger/David J. Krieger (Hg.), ANThology. Ein einführendes Handbuch zur Akteur-Netzwerk-Theorie, Bielefeld, 2006, S. 135-174: 143.

10 Bublitz/Marek/Steinmann/Winkler (2010), Einleitung, S. 9 und S. 12.

11 Ebd., S. 9.

12 Hartmut Winkler, „Es gibt eine spezifische Opazität des Handelns, und Handlungen haben unintendierte Folgen“, in: Hannelore Bublitz/Roman Marek/Christina L. Steinmann/ders. (Hg.), Automatismen, München, 2010, S. 110-113: 110 f. 
den Handlungsquellen, die man eine nach der anderen $\mathrm{zu}$ entwirren lernen $\mathrm{mu}$. $^{13}$

Die Aufgabe, die von Latour hier beschrieben wird, ließe sich - in Fortsetzung der Metapher des Seils, also von ,Knoten“ und ,Schlinge', die es zu ,entwirren“ gilt - auch beschreiben als eine „Strukturbeschreibung durch Entflechtung". Damit erscheint die ANT als vielversprechende Kandidatin auf der Suche nach methodischen Instrumentarien und theoretischen Begriffen für die Analyse der Entstehung ungeplanter Strukturen. ${ }^{14}$

Gleichwohl ergibt sich mit Blick auf das Handlungsverständnis auch eine mögliche Spannung zwischen Automatismen-Konzept und Prämissen der ANT: Zur Beschreibung der Emergenz ungeplanter Strukturen wird in der Analyse von Automatismen die Metaphorik verwendet, dass sich die emergierenden Strukturen ,im Rücken der beteiligten Akteure ausbilden ${ }^{6}{ }^{15}$ Die ANT hingegen hebt hervor, dass sich Handeln zwar der bewussten Kontrolle Einzelner entzieht, aber gleichzeitig nichts im Hintergrund, im Rücken der Akteure geschehe. So betont Latour in der Neuen Soziologie:

Die Gesetze der sozialen Welt mögen existieren, doch sie nehmen eine ganz andere Position ein, als die Tradition zunächst gedacht hatte. Sie befinden sich nicht hinter der Bühne, nicht über den Köpfen oder vor der Handlung, sondern nach der Handlung, unter den Teilnehmern und ganz im Vordergrund. ${ }^{16}$

Latour geht es hier offensichtlich darum, jedweden reduktionistischen Erklärungen eine Absage zu erteilen. Er wendet sich damit gegen Ansätze, die das Handeln sozialer Akteure auf bestimmte vorgelagerte und determinierende Ursachen zurückführen ${ }^{17}$; gegen ein ,Mastermind", das aber selbst im Hintergrund bleibt und mit unsichtbarer - aber planender - Hand das Schicksal der involvierten Akteure bestimmt. ${ }^{18}$

In der Erklärung offenbart sich aber zugleich, dass es sich eher um eine Spannung in der Rhetorik als in der Sache handelt, denn auch im Automatismen-Konzept geht es schließlich gerade darum, traditionelle, reduktionistische Erklärungen zu überwinden. So wird auch hier hervorgehoben, dass Automatismen sich nicht ,auf eine im Voraus berechenbare Rationalität [...] reduzie-

13 Latour (2007), Eine neue Soziologie, S. 77. [Herv. T. C./H. D./F. M.]

14 Eine offene Frage bleibt diesbezüglich, welche Strukturprinzipien durch das Diktum, Strukturen könnten nur in ihrer Konstruktion nachvollzogen, nicht aber als ,soziale Asymmetrien betrachtet werden, möglicherweise aus dem Blick geraten. Vgl. Latour (2007), Eine neue Soziologie, S. 111.

15 Bublitz/Marek/Steinmann/Winkler (2010), Einleitung, S. 9.

16 Latour (2007), Eine neue Soziologie, S. 422 f.

17 In der Ablehnung einer solchen Perspektive zeigt sich das ethnomethodologische Erbe der ANT. Diese betont mit dem Konzept der ,Vollzugswirklichkeit', dass alle Handlungen in der Gegenwart vollzogen werden. Es existieren keine Strukturen, die nicht im gegenwärtigen Moment (re)produziert werden.

18 Im Essay Das Elend der Kritik. Vom Krieg um Fakten zu Dingen von Belang, Zürich, Berlin, 2007 [engl. OA 2004], S. 14-17, geht Latour so weit, solche Erklärungen in die Nähe von Verschwörungstheorien zu rücken. 
ren lassen“"19. Der Frage nach Automatismen geht es - den Bemühungen der ANT vergleichbar - gerade darum, Komplexität nicht im Vorfeld einer Analyse durch einen Rückgriff auf zentralistische top down gerichtete Erklärungen einzuschränken. ${ }^{20}$ Damit zeichnet sich in der Frage nach der Reduzierung von Komplexität eine weitere Strukturähnlichkeit zwischen dem Konzept der Automatismen und dem Akteur-Netzwerk-Ansatz ab. Dies betrifft im Besonderen das Moment der Genese von Automatismen: Entsprechend der Annahme einer Opazität des Handelns entwickeln sich Automatismen dort, wo - beispielsweise durch Gewöhnung, Training oder Einschreibung - das Bewusstsein weitestgehend ausgeschaltet wird. ${ }^{21}$ Eine mit steter Regelmäßigkeit ausgeführte Handlung bedarf $a b$ einem gewissen Moment keiner weiteren Anstrengung mehr und gerinnt durch den Mechanismus der Wiederholung selbst zum Automatismus. ${ }^{22}$

Diese Erkenntnis findet sich auch im Begriff der ,Blackbox‘ oder, wie John Law es nennt, der ,Punktualisierung ' wieder:

In der Praxis haben wir es nicht mit endlosen Netzwerkverzweigungen zu tun; tatsächlich können wir meistens noch nicht einmal die Komplexität von Netzwerken wahrnehmen. [...] Falls ein Netzwerk als einziger Block handelt, verschwindet es, um von der Handlung selbst und dem anscheinend simplen Autor dieser Handlung ersetzt zu werden. Gleichzeitig wird die Art, in der der Effekt erzeugt wird, gelöscht: Zum gegebenen Zeitpunkt ist sie weder sichtbar noch relevant. Auf diese Weise maskiert zu bestimmten Zeiten ein einfacheres Element - ein funktionierendes Fernsehgerät, eine gut verwaltete Bank oder ein gesunder Körper - das es produzierende Netzwerk. ${ }^{23}$

Auch in diesem Punkt scheinen ANT und Automatismenforschung also ein ähnliches Anliegen zu teilen: Ziel von Analysen ist es, ,Blackboxes‘ (bzw. so

19 Bublitz/Marek/Steinmann/Winkler (2010), Einleitung, S. 10.

20 MarionettenspielerInnen, die im Hintergrund die Fäden ziehen, kann es dementsprechend in keinem der beiden Ansätze geben. Das entsprechende Bild wären - für die Automatismen, wie auch in der ANT - vielmehr MarionettenspielerInnen, die glauben eine bestimmte Figur hinter ihrem Rücken zu lenken, ohne dabei die multivarianten Beziehungen innerhalb des Puppentheaters wahrzunehmen, zu beachten oder auch nur in Betracht zu ziehen. Vgl. John Law, „Actor Network Theory and Material Semiotics“, in: Bryan S. Turner (Hg.), The New Blackwell Companion to Social Theory, Malden, MA, 2009, S. 141-158: 151. Siehe auch die deutsche Übersetzung in diesem Band.

21 Vgl. Hannelore Bublitz, ,Täuschend natürlich. Zur Dynamik gesellschaftlicher Automatismen, ihrer Ereignishaftigkeit und strukturbildenden Kraft", in: dies./Roman Marek/Christina L. Steinmann/Hartmut Winkler (Hg.), Automatismen, München, 2010, S. 153-171 sowie dies., ,Automatismen beinhalten einen qualitativen Sprung: Aus der wiederholten Einschleifung durch Übung entsteht - paradoxerweise - gerade das Neue: spielerisch-mühelose Perfektion“, in: dies./Roman Marek/Christina L. Steinmann/Hartmut Winkler (Hg.), Automatismen, München, 2010, S. 23-26.

22 Vgl. hierzu auch: Hartmut Winkler, Diskursökonomie. Versuch über die innere Ökonomie der Medien, Frankfurt/M., 2004, S. 192.

23 John Law, „Notizen zur Akteur-Netzwerk-Theorie: Ordnung, Strategie und Heterogenität“ [engl. OA 1992], in: Andréa Belliger/David J. Krieger (Hg.), ANThology. Ein einführendes Handbuch in die Akteur-Netzwerk-Theorie, Bielefeld, 2006, S. 429-446: 436. 
verstandene Automatismen) zu öffnen und deren Entstehungsprozesse und Mechanismen zu rekonstruieren.

Selbstverständlich ist es keine einfache Aufgabe, die (materialen) Verbindungen und die Vielzahl (überraschender) Entitäten nachzuzeichnen, die Handeln und Strukturbildung ermöglichen. Aber die ANT kann mit ihren Fallstudien und methodologischen Überlegungen impulsgebend für die Automatismenforschung sein. Sie steht beispielhaft für eine Analyseperspektive, die sich bemüht, die einzelnen Schritte und die Arbeiten, die notwendig sind, um Strukturen hervorzubringen, nachzeichnen und beschreiben zu können. $\mathrm{Zu}$ gleich kann sie auch als Warnung angesehen werden, sich nicht in einen unhaltbaren Holismus und in vorschnelle Erklärungen zu verstricken.

Insgesamt zeigt sich also, dass die Auseinandersetzung mit der ANT der Automatismenforschung wichtige Denkanreize liefert, die zur Schärfung und Operationalisierung des Automatismen-Konzeptes beitragen können; nicht weil sie den einen Weg zur Untersuchung ungeplanter Strukturentstehung aufzeigt, sondern weil sie, wie eben gezeigt, bestimmte methodische Anregungen und beispielhafte Studien liefert. Diese bieten Reibungsflächen und regen zum Nach-Denken (und vielleicht auch Neu-Denken) über etablierte sozial- und kulturwissenschaftliche Grenzziehungen an - und damit auch zur Klärung der eigenen Sichtweise. Wir hoffen, dass dies in den vielfältigen Beiträgen dieses Bandes zum Ausdruck kommt und den LeserInnen entsprechend Anregungen zur Reflexion und zum Weiterdenken der eigenen Forschungspraxis geliefert werden.

\section{Aufbau des Bandes}

Den Auftakt macht ein einleitender Text von John Law, in dem er Geschichte und Entwicklung der ANT in den vergangenen 30 Jahren darstellt. Sein Beitrag gibt einen Überblick über die heterogene, fachlich und geografisch dezentrierte ,Gemeinschaft' der Akteur-Netzwerk-Theorie(n). Die nachfolgenden Beiträge sind nach drei thematischen Schwerpunkten geordnet. Im ersten Teil werden, ausgehend vom Symmetriepostulat der ANT, überraschende Handlungsquellen in den Blick genommen, die das Spektrum der an Strukturbildung beteiligten Akteure erweitern. Davon ausgehend verschiebt sich die Perspektive im zweiten Teil auf Strukturprinzipien und die Frage nach der Rolle von Macht in Akteur-Netzwerken. Der dritte Abschnitt widmet sich Akzentverschiebungen, Kritiken und Kontroversen, die in der (inter-)disziplinären Auseinandersetzung mit der ANT entstehen. 


\section{Überraschende Handlungsquellen}

Das verallgemeinerte Symmetrieprinzip und die damit einhergehende Assoziierung von Menschen und nicht-menschlichen Akteuren führt zu neuen Anordnungen gängiger Forschungsgegenstände, wie die ersten Beiträge zu überraschenden Handlungsquellen zeigen. Diese versammeln Fallstudien, die untersuchen, wie sich die Betrachtung sozialer und räumlicher Ordnungen durch die symmetrische Perspektive auf heterogene Akteur-Netzwerke verändert. Das überraschende Moment liegt in der Wahrnehmung von Akteuren, deren Ausdehnung und Handlungsmacht vorher randständig oder unsichtbar blieb. Durch das Symmetrieprinzip geraten sie in den Blick der Forschenden: ein Virus in der Soziologie, ,Unbekannte' in der Quantenphysik, Atmosphären in der Architektur und Kulturwissenschaft und wissenschaftliche Arbeitsweisen in der zeitgenössischen Kunst.

Im ersten Beitrag fragen Wiebke Pohler und Michael Schillmeier aus einer soziologischen Perspektive, wie das SARS-Virus als grenzüberschreitender öffentlicher Akteur vorhandene gesellschaftliche Ordnungsmuster verändert. Sie beschreiben ein zirkulierendes heterogenes Netzwerk aus Viren, Zellen, Menschen, Tieren und Technologien als kosmo-politisches Ereignis, das Gebiete der öffentlichen Gesundheitssysteme, der Wissenschaft und der Rechte beeinflusst hat.

Julian Rohrhuber begibt sich mit seinem Beitrag zu Operatoren und Operationen in die ANT-geleitete Analyse wissenschaftlicher Praxis. Das Konzept der von Latour beschriebenen operativen Ketten und die daran geknüpfte Vorstellung von Medialität werden problematisch, wenn wie in der Quantenphysik ,Unbekannte“ Teil der Operationsketten werden.

Als unbestimmter und zudem flüchtiger Akteur erweisen sich auch Atmosphären, die in den Beiträgen von Anke Rees und Christoph Michels in der Verbindung mit Architektur und räumlichen Ordnungsweisen bearbeitet werden. Anke Rees widmet sich den Kontroversen um die Hamburger Schilleroper, die sie als teils dauerhaftes, teils vergängliches Netzwerk aus Materialien und menschlichen sowie tierischen NutzerInnen des Gebäudes begreift. Die Veränderungen des Netzwerkes erzeugen bestimmte Atmosphären, die Einfluss auf Aushandlungsprozesse in der Stadtentwicklung nehmen. Christoph Michels sieht in Atmosphären das Potenzial zu Emanzipation und kreativer Veränderungsmacht. Am Beispiel der urbanen Sportart Parkour zeigt er wie Ordnungsweisen die Herstellung von Atmosphären durch Körper und Umwelt regulieren.

Vor dem Hintergrund der Arbeiten Latours befragt Renate Wieser zwei Kunstwerke, wie die Annäherung von Kunst und Wissenschaft aussehen beziehungsweise deren Gegenüberstellung überwunden werden kann. Die beiden Werke inszenieren jeweils in einer Laborsituation Forschungen zur Evolutionsbiologie und Künstlicher Intelligenz, um die Grenzziehungen zwischen naturwissenschaftlicher und kultureller Handlungsmacht auszutesten. 


\section{Macht in Akteur-Netzwerken}

Die Beiträge des zweiten Abschnitts widmen sich Fragen der Konzeption von Macht in Akteur-Netzwerken. Zumindest die ,klassische“ ANT erweist sich hinsichtlich dieser Fragen als ambivalent. Finden sich in konzeptionellen Texten einige Bezüge beispielsweise auf die Arbeiten Foucaults ${ }^{24}$, bleiben diese in konkreten Fallstudien meist wenig beachtet. ${ }^{25}$ Hier setzen die Texte dieses Abschnitts an und stellen Reflexionen über einen Typus der Macht in den Mittelpunkt, der weder auf eine singuläre, ausübende Instanz zurückverweist noch top down und zentralistisch im Modus der Souveränität operiert. Das Interesse gilt den Machtwirkungen, deren Hauptaufgabe darin besteht, Verbindungen innerhalb eines ,heterogenen Ensembles ${ }^{\varsigma}$ zu organisieren. Foucault erscheint als grundlegende Referenz für die Analyse von Machtbeziehungen in AkteurNetzwerken. Gleichzeitig erweist er sich als entscheidender Mittler, wenn es darum geht, Fragen nach Automatismen mit den theoretischen Perspektiven des Akteur-Netzwerk-Ansatzes zu verflechten. Wesentlich ist hierfür, dass Macht nicht als Ursache, sondern als temporär stabilisierender Effekt in der Etablierung ungeplanter Strukturen gedacht wird: „Die Macht ist nicht etwas, was man erwirbt, wegnimmt, teilt, was man bewahrt oder verliert; die Macht ist etwas, was sich von unzähligen Punkten aus und im Spiel ungleicher und beweglicher Beziehungen vollzieht.“26

Im ersten Beitrag betrachtet Andrea Seier das Verhältnis von Foucaults Dispositiven zu Latours Agenturen aus medienwissenschaftlicher Perspektive. Sie kann aufzeigen, dass sich beide durchaus erfolgreich aufeinander beziehen lassen, ohne dass die Differenzen zwischen den Ansätzen aus dem Blick geraten müssen. Sowohl Dispositive als auch Agenturen ermöglichen es demnach, Medien zugleich als Resultat und Produzent verteilter Handlungsmacht zu analysieren. Die besondere Aufmerksamkeit der ANT für die Rolle nichthumaner Akteure kann die Analyse von Macht/Wissen-Strukturen und SelbstTechnologien konkretisieren und sinnvoll ergänzen.

Ausgehend von den relationalen Machtmodellen Foucaults und der ANT diskutiert Theo Röhle die Frage nach der Funktion von Intentionalität für die Entstehung ungeplanter Strukturen und die Stabilisierung von Machtrelationen. Sowohl Foucault als auch die ANT verorten Machtwirkungen jenseits individueller Interessen und beschreiben Formen nicht-subjektiver Intentionalität. Foucault zufolge basiert diese auf der Trennung zwischen einer taktischen und einer strategischen Ebene, die in ihrem rekursiven Zusammenspiel Koordination und Dynamik generieren. In der ANT, die sich eher für lokale Interaktionen interessiert, erscheint die Frage nach Intentionalität als besondere

24 Vgl. bspw. Law (2006), Notizen zur Akteur-Netzwerk-Theorie, S. 438. Siehe auch Latour (2007), Eine neue Soziologie, S. 148.

25 Vgl. hierzu Law (2009), Actor Network Theory and Material Semiotics, S. 154.

26 Michel Foucault, Der Wille zum Wissen. Sexualität und Wahrheit 1, 14. durchgesehene und korrigierte Aufl., Frankfurt/M., 1983 [frz. OA 1976], S. 94. 
Herausforderung an eine adäquate Beschreibungssprache in konkreten Analysen.

Im Rahmen einer ausführlichen Fallstudie sucht Anne Dölemeyer nach Möglichkeiten, Aspekte der ANT mit Elementen Foucault'scher Gouvernementalitätsanalysen zu verbinden. In ihrer Analyse der Planungsprozesse zum Wiederaufbau des durch den Hurrikan Katrina zerstörten New Orleans zeigt sie Möglichkeiten und Grenzen einer solchen Perspektive für Fragestellungen politischer Repräsentation und Modi des Regierens auf. Karten und Daten werden innerhalb eines weit verzweigten Governance-Netzwerks zu entscheidenden Akteuren im Kampf um (Handlungs-)Macht.

In der Fallstudie von Thomas Foth geht es um die systematische Ermordung von Kranken im Nationalsozialismus und speziell um den Akteursstatus von PatientInnenakten in dieser Maschinerie. Er kombiniert Latours Konzepte der inscription devices und immutable mobiles mit einem ebenfalls an Foucault orientierten diskursanalytischen Ansatz. Dadurch zeigt Foth, dass die Akte innerhalb des komplexen Netzwerks psychiatrischer Tötungsanstalten des NS-Regimes eine tragende Rolle für die Konstruktion der Identität von PatientInnen spielt - und deren Auslöschung gleichzeitig aktiv mit vorbereitet.

\section{Akzentverschiebungen, Kritik und Kontroversen}

Im dritten und abschließenden Teil Akzentverschiebungen, Kritik und Kontroversen sind Beiträge versammelt, die in der ANT verhandelte Begriffe und Konzepte aufgreifen, um an diese anknüpfend Diskussionen aufzunehmen und weiterzuführen. So geht es um Latours immutable mobiles, das Symmetriekonzept, den Begriff des Politischen und das Verhältnis der ANT zu kritischen Ansätzen in den Sozial- und Kulturwissenschaften. Gemeinsam ist allen Texten, dass sie die ANT nicht als feststehendes Theoriegebäude begreifen, sondern Korrekturen und Verschiebungen vornehmen, die für eigene Fragestellungen produktiv gemacht werden und zur Schärfung der je eigenen Begrifflichkeiten dienen.

Den Auftakt macht der Beitrag von Erhard Schüttpelz. Dieser fragt, wie eine Geschichte moderner Medien nach Maßgabe der ANT vorgehen kann. Am Beispiel von Latours Analyse der Entwicklung der Kodak-Kamera zeigt er, dass Medienentwicklung als nicht-planbarer Prozess betrachtet werden muss, der sich aus - teils widerstreitenden - technischen, natürlichen, sozialen und diskursiven Ursachen zusammensetzt. Über Latours Konzept der immutable mobiles führt ihn dieser Gedanke zu einer revidierten Darstellung der Entwicklung moderner Medien.

Der daran anschließende Beitrag von Jan-Hendrik Passoth bringt ,postklassische' Praxistheorien innerhalb der Soziologie mit der Akteur-NetzwerkTheorie ins Gespräch. Auf diese Weise sollen zum einen die speziellen Gegenstände der STS näher an die Soziologie herangerückt werden. Zum ande- 
Bublitz, Hannelore/Marek, Roman/Steinmann, Christina L./Winkler, Hartmut, „Einleitung“, in: dies. (Hg.), Automatismen, München, 2010, S. 9-16.

Bublitz, Hannelore, ,Automatismen beinhalten einen qualitativen Sprung: Aus der wiederholten Einschleifung durch Übung entsteht - paradoxerweise - gerade das Neue: spielerisch-mühelose Perfektion“, in: dies./Roman Marek/Christina L. Steinmann/Hartmut Winkler (Hg.), Automatismen, München, 2010, S. 23-26.

Dies., „Täuschend natürlich. Zur Dynamik gesellschaftlicher Automatismen, ihrer Ereignishaftigkeit und strukturbildenden Kraft", in: dies./Roman Marek/Christina L. Steinmann/Hartmut Winkler (Hg.), Automatismen, München, 2010, S. 153-171.

Callon, Michel, „Einige Elemente einer Soziologie der Übersetzung: Die Domestikation der Kammmuscheln und der Fischer der St. Brieuc-Bucht", in: Andréa Belliger/David J. Krieger (Hg.), ANThology. Ein einführendes Handbuch zur AkteurNetzwerk-Theorie, Bielefeld, 2006, S. 135-174. [Engl. OA 1986.]

Foucault, Michel, Der Wille zum Wissen. Sexualität und Wahrheit 1, 14. durchgesehene und korrigierte Aufl., Frankfurt/M., 1983. [Frz. OA 1976.]

Gießmann, Sebastian/Brunotte, Ulrike/Mauelshagen, Franz/Böhme, Hartmut/Wulf, Christoph (Hg.), Zeitschrift für Kulturwissenschaften. Politische Ökologie, Bielefeld, 2 (2009).

Kneer, Georg/Schroer, Markus/Schüttpelz, Erhard (Hg.), Bruno Latours Kollektive. Kontroversen zur Entgrenzung des Sozialen, Frankfurt/M., 2008.

Latour, Bruno, Eine neue Soziologie für eine neue Gesellschaft. Einführung in die Akteur-Netzwerk-Theorie, Frankfurt/M., 2007. [Engl. OA 2005.]

Ders., Das Elend der Kritik. Vom Krieg um Fakten zu Dingen von Belang, Zürich, Berlin, 2007. [Engl. OA 2004.]

Law, John, „Actor Network Theory and Material Semiotics“, in: Bryan S. Turner (Hg.), The New Blackwell Companion to Social Theory, Malden, MA, 2009, S. 141158.

Ders., „Notizen zur Akteur-Netzwerk-Theorie: Ordnung, Strategie und Heterogenität“", in: Andréa Belliger/David J. Krieger (Hg.), ANThology. Ein einführendes Handbuch in die Akteur-Netzwerk-Theorie, Bielefeld, 2006, S. 429-446. [Engl. OA 1992.]

Ders./Hassard, John (Hg.), Actor Network Theory and After, Oxford (u. a.), 1999.

Winkler, Hartmut, „Es gibt eine spezifische Opazität des Handelns, und Handlungen haben unintendierte Folgen", in: Hannelore Bublitz/Roman Marek/Christina L. Steinmann/ders. (Hg.), Automatismen, München, 2010, S. 110-113.

Ders., Diskursökonomie. Versuch über die innere Ökonomie der Medien, Frankfurt/M., 2004. 
Tobias Conradi, Heike Derwanz, Florian Muhle (Hrsg.)

STRUKTURENTSTEHUNG DURCH VERFLECHTUNG 


\section{„AUTOMATISMEN“}

Herausgegeben von

Hannelore Bublitz, Gisela Ecker,

Norbert Eke, Reinhard Keil

und Hartmut Winkler 
Tobias Conradi, Heike Derwanz, Florian Muhle (Hrsg.)

\section{STRUKTURENTSTEHUNG DURCH VERFLECHTUNG}

Akteur-Netzwerk-Theorie(n) und Automatismen

Wilhelm Fink 
Gedruckt mit Unterstützung der Deutschen Forschungsgemeinschaft

Umschlagabbildung:

Jürgen Gebhard (picturepress)

Bibliografische Information der Deutschen Nationalbibliothek

Die Deutsche Nationalbibliothek verzeichnet diese Publikation in der Deutschen Nationalbibliografie; detaillierte bibliografische Daten sind im Internet über http://dnb.d-nb.de abrufbar.

Gedruckt auf umweltfreundlichem, chlorfrei gebleichtem und alterungsbeständigem Papier.

Alle Rechte, auch die des auszugsweisen Nachdrucks, der fotomechanischen Wiedergabe und der Übersetzung, vorbehalten. Dies betrifft auch die Vervielfältigung und Übertragung einzelner Textabschnitte, Zeichnungen oder Bilder durch alle Verfahren wie Speicherung und Übertragung auf Papier, Transparente, Filme, Bänder, Platten und andere Medien, soweit es nicht

$\S \S 53$ und 54 UrhG ausdrücklich gestatten.

(C) 2011 Wilhelm Fink Verlag, München

(Wilhelm Fink GmbH \& Co. Verlags-KG, Jühenplatz 1, D-33098 Paderborn)

Internet: www.fink.de

Lektorat und Satz: Margret Westerwinter, Düsseldorf

Einband: Evelyn Ziegler, München

Printed in Germany.

Herstellung: Ferdinand Schöningh GmbH \& Co. KG, Paderborn

ISBN 978-3-7705-5220-7 


\section{INHALT}

Tobias ConRadi, Heike Derwanz, Florian MuHLE

Strukturentstehung durch Verflechtung - Zur Einleitung ........... 9

JOHN LAW

Akteur-Netzwerk-Theorie und materiale Semiotik

\section{ÜBERRASCHENDE HANDLUNGSQUELLEN}

MichAel SCHILLMEIER, WIEBKe POHLER

Topologie von (Un-)Sicherheitsstrukturen.

Zur Kosmo-Politik viraler Infektionen

JULIAN ROHRHUBER

Operation, Operator - Sehen, was das Photon sieht

ANKE REES

Widerspenstige Gebäude.

Eine Untersuchung von Materialität, Kontroversen

und Atmosphären

CHRISTOPH Michels

Parkour erleben:

Wie Atmosphären inszeniert werden

RENATE WIESER

Labore der Kunst.

Über unmögliche Anatomie und einen Milchglas-Fetisch 


\section{MACHT IN AKTEUR-NETZWERKEN}

ANDREA SEIER

Un/Verträglichkeiten:

Latours Agenturen und Foucaults Dispositive

THEO RÖHLE

Strategien ohne Strategen.

Intentionalität als „Strukturentstehung durch Verflechtung“? .... 173

ANNE DÖLEMEYER

Re-Membering New Orleans. Planung, Partizipation

und Repräsentation nach Katrina

THOMAS FOTH

Regieren durch Akten. Die Funktion von PatientInnenakten

für die Krankenmorde des Nationalsozialismus

\section{AKZENTVERSCHIEBUNGEN, KRITIK UND KONTROVERSEN}

ERHARD SCHÜTTPELZ

Moderne Medien ohne Modernisierungstheorie 239

JAN-HENDRIK PASSOTH

Fragmentierung, Multiplizität und Symmetrie.

Praxistheorien in post-pluraler Attitüde

DOMINIQUE RUDIN

Sozialität und Konflikt mit der

Akteur-Netzwerk-Theorie denken:

Skizze einer Heuristik aus historischer Perspektive

279 
KATHARINA HOLAS

Technoscience: Akteur-Netzwerk-Theorien und feministische Akzentverschiebungen

TOBIAS CONRADI, FLORIAN MUHLE

Verbinden oder trennen? Über das schwierige Verhältnis der Akteur-Netzwerk-Theorie zur Kritik

ABBILDUNGSNACHWEISE 335

ÜBER DIE AUTORINNEN UND AUTOREN 337 